Chapman University

Chapman University Digital Commons

Economics Faculty Articles and Research

Economics

2012

\title{
Coalitional Colonel Blotto Games with Application to the Economics of Alliances
}

Dan Kovenock

Chapman University, kovenock@chapman.edu

Brian Roberson

Purdue University

Follow this and additional works at: http://digitalcommons.chapman.edu/economics_articles

Part of the Economics Commons, and the Military Studies Commons

\section{Recommended Citation}

Kovenock, Dan, and Brian Roberson. "Coalitional Colonel Blotto Games with Application to the Economics of Alliances." Journal of Public Economic Theory 14 (2012): 653-676.

DOI:10.1111/j.1467-9779.2012.01556.x

This Article is brought to you for free and open access by the Economics at Chapman University Digital Commons. It has been accepted for inclusion in Economics Faculty Articles and Research by an authorized administrator of Chapman University Digital Commons. For more information, please contact laughtin@chapman.edu. 


\section{Coalitional Colonel Blotto Games with Application to the Economics of}

Alliances

\section{Comments}

This is a pre-copy-editing, author-produced PDF of an article accepted for publication in Journal of Public Economic Theory, 14, 2012 following peer review. The final publication is available at Springer via DOI: $10.1111 /$ j.1467-9779.2012.01556.x.

\section{Copyright}

Springer 


\title{
Coalitional Colonel Blotto Games with Application to
}

\section{the Economics of Alliances ${ }^{1}$}

\author{
Dan Kovenock ${ }^{2}$ and Brian Roberson ${ }^{3}$
}

\begin{abstract}
${ }^{1}$ We have benefited from the helpful comments of Daniel G. Arce, Timothy Feddersen, Steven Slutsky, Konstantin Sonin, and participants in presentations at the Social Science Research Center Berlin (WZB), the 2006 Annual Meeting of the Midwest Political Science Association, the First World Meeting of the Public Choice Society, the Eighth Conference of the Society for the Advancement of Economic Theory, the 2007 Annual Meeting of the American Political Science Association, and the 2007 Annual Meeting of the Southern Economic Association. Part of this work was completed while Kovenock was Visiting Professor at the Social Science Research Center Berlin (WZB). Roberson acknowledges financial support from the Miami University Committee on Faculty Research and the Farmer School of Business. The authors, of course, remain solely responsible for any errors or omissions.

${ }^{2}$ Dan Kovenock, University of Iowa, Department of Economics, Tippie College of Business, W284 PBB, 21 E. Market Street, Iowa City, IA 52242 USA t: 319-335-1810, E-mail: dankovenock@uiowa.edu

${ }^{3}$ Brian Roberson, Purdue University, Department of Economics, Krannert School of Management, 403 W. State Street, West Lafayette, IN 47907 USA t:765-494-4531 E-mail: brobers@purdue.edu (Correspondent)
\end{abstract}




\begin{abstract}
In Borel's (1921) Colonel Blotto game two players simultaneously allocate their respective endowments of a resource across $n$ battlefields, the higher allocation wins each battlefield, and players maximize the number of battlefields won. Here we examine two players who may form an alliance before separately competing in two disjoint Colonel Blotto games against a common adversary. Despite a lack of common interests, unilateral transfers — in a direction consistent with the exploitation hypothesis - arise for a range of parameter configurations. Such transfers alter the adversary's strategy and the combination of the direct and strategic effects benefits both allies.
\end{abstract}




\section{Introduction}

The Colonel Blotto game examines the strategic allocation of resources across a set of battlefields. In the original formulation, due to Borel (1921), two players allocate their respective endowments of a resource across a finite number of battlefields. Each player must distribute their resource without knowing their opponent's distribution of the resource and the player who submits the higher level of the resource to a battlefield wins that battlefield. Each player's payoff for the whole game is the sum of his wins across the individual battlefields. The Colonel Blotto game figured prominently in the early game theory literature and applications of the model in public economics and political economy include the analyses of multi-battle military conflict, electoral campaign resource allocation, and redistributive political competition. ${ }^{1}$

This paper examines a multi-player, multi-front Colonel Blotto game in which one player, $A$, simultaneously competes in two disjoint Colonel Blotto games, against two separate opponents, 1 and 2. Prior to competing in the games, players 1 and 2 have the opportunity to form an alliance to share their endowments of a one-dimensional resource (e.g., troops, military hardware, money). Our focus is on non-cooperative alliances in which only individually rational ex ante transfers of the resource are allowed. Once these transfers take place, player A optimally responds in allocating his resource endowment across the two games and then players play their respective Colonel Blotto games given their resource constraints. No ex post transfers between the two alliance members are enforceable. We call such an alliance a self-enforcing alliance without commitment.

\footnotetext{
${ }^{1}$ Early contributions include Borel and Ville (1938), Tukey (1949), Gross and Wagner (1950), Gross (1951), Blackett $(1954,1958)$, and Bellman (1969). More recently, work on Blotto-type games has examined asymmetries between the players (Hart 2008, Macdonell and Mastronardi 2010, Roberson 2006, Weinstein 2005), non-constant-sum variations (Kvasov 2007, Hortala-Vallve and Llorente-Saguer 2010a, b, Roberson and Kvasov 2008), alternative definitions of success (Golman and Page 2009, Szentes and Rosenthal 2003a, b, Kovenock and Roberson 2010, and Tang, Shoham, and Lin 2010), and political economy applications (Laslier 2002, Laslier and Picard 2002, Roberson 2008).
} 
The main result of this paper is to show that, even though each member of the alliance shares no common interest in the outcome of the other member's game, there is a wide range of parameters in which endogenous unilateral transfers take place within such an alliance. That is, one player gives away resources to its ally, who happily accepts the gift. Unilateral transfers arise because they lead to a strategic shift in the common opponent's force allocation away from the set of battlefields common to the player making the transfer, towards the set of battlefields common to the player receiving the transfer. Our result demonstrates that there exist unilateral transfers for which the combination of the direct and strategic effects benefits both allies.

Our approach contrasts with the major focus of the literature on the economics of alliances, dating back to Olson (1965) and Olson and Zeckhauser (1966), (for a summary, see Sandler and Hartley, 2001). This literature generally assumes that the resource employed by allies is a (possibly impure) public good. ${ }^{2}$ In these models, one player's resource allocation provides direct non-rival, non-excludable benefits to an allied player. ${ }^{3}$ In our model, resource expenditure by an ally is completely rival and excludable. However, through its effects on the strategic choices of the enemy, strategic externalities may be created. These externalities may suffice to generate endogenous unilateral transfers in strategic alliances without the $a$ priori assumption of pure or impure public goods and without commitment.

\footnotetext{
${ }^{2}$ In Olson (1965) and Olson and Zeckhauser (1966) alliance expenditure was treated as a pure public good. Extensions to impure public good expenditure, known as the "joint product model" originate with Van Ypersele De Strihou (1967). See also Sandler and Cauley (1975), Sandler (1977), Murdoch and Sandler (1982, 1984), and Ihori and McGuire (2007).

${ }^{3}$ In early contributions to this literature it was standard to focus solely on the game between alliance members and take the enemys expenditure as given. Exceptions to this approach include Bruce (1990), Linster (1993), and Skaperdas (1998). Bruce examines a model of pure public good expenditure in which "security" enters directly as a good into the utility function and is measured by the difference between total allied expenditure and enemy expenditure. His assumptions generate upward sloping best response functions in expenditures. Linster (1993) and Skaperdas (1998) examine the formation of alliances in contests in which the probability of winning a prize is represented by a contest success function and the expenditure of each alliance member serves as a (possibly impure) public good in that it directly increases the expected payoffs of other alliance members for a given enemy expenditure in the contest. For surveys of the literature utilizing this and similar approaches see Bloch (2009) and Konrad (2009).
} 
Despite its significant departure from the assumptions of the public goods-based literature on alliances, our model also obtains results consistent with one prominent conjecture in that literature, Olson's (1965) "exploitation hypothesis." This hypothesis asserts that larger nations will bear a disproportionately higher share of the common cost of an alliance relative to its benefits. In our model, a self-enforcing alliance without commitment arises involving unilateral transfers from player $i$ to player $j$ when player $i$ has a larger resource endowment and the ratio of player $i$ 's endowment to player $j$ 's endowment is sufficiently greater than the ratio of the total values of the battlefields in the two players' respective Colonel Blotto games. When such alliances arise, transfers flow from the player who is resource rich to the player who is resource poor. The degree of asymmetry in resource endowments necessary to generate a self-enforcing alliance without commitment depends not only on the relative aggregate values of the players' respective battlefields, but also on the absolute magnitudes of the two players' endowments relative to that of player $A$.

Our model appears to provide potential insight into the formulation of nations' foreign policies with respect to defense and strategic alliances as well as the behavior of alliances in historical military conflicts. Often such alliances involve powerful nations providing material or financial support to other nations whose values are very much at odds with their own. The motivation for such action is often described by the phrase, "the enemy of my enemy is my friend." During the Cold War the United States supported dictators in Zaire and Chile and the Afgan Mujahideen. ${ }^{4}$ Similarly, the Soviet Union supported strongly anti-Communist governments such as the Nasser regime in Egypt. After the Iranian Revolution of 1979 the United States provided aid to Saddam Hussein in the Iran-Iraq War. Chinese support for Pakistan has been attributed to its desire to divert Indian resources from their own rivalry. ${ }^{5}$

Our approach also appears useful in explaining the assistance that the United States

\footnotetext{
${ }^{4}$ For further details see the political science literature on human rights and US military aid (e.g. Meernik, Krueger, and Poe 1998 or Cingranelli and Pasquarello 1985).

${ }^{5}$ See Hoey (2009) for further details.
} 
provided to the Soviet Union in The Second World War through the Lend-Lease Act of 1941. Although the United States provided approximately 50 billion dollars aid to a host of countries, including Britain, France, and China, transfers to the Soviets took place despite a strong adversarial relationship between the nations. ${ }^{6}$ Estimates of these transfers vary, ranging from $\$ 9$ Billion to $\$ 11$ billion for the four-year period after Nazi Germany's invasion of the Soviet Union in 1941. Historical accounts of this program lend some support for the view that this assistance was extended with no expectation of repayment. ${ }^{7}$

Section 2 introduces our three stage game. Section 3 examines equilibrium in the final stage of the game, which consists of a multi-player, multi-front Colonel Blotto game in which one player, $A$, simultaneously competes in two disjoint Colonel Blotto games, against two separate opponents, 1 and 2 . This section provides a characterization of the payoffs of the component Colonel Blotto games for arbitrary budget constraints and any number of battlefields $n \geq 3$. The resource endowments in this final stage are determined by choices made in the first two stages. These two stages are examined in Section 4. In the first stage, conditional on their endowments, players 1 and 2 decide on whether to transfer resources, with any positive net transfer generating a self-enforcing alliance without commitment. In the second stage, player $A$ decides upon an allocation of its resources across the two Blotto games, contingent on the choices of players 1 and 2. Section 4 shows that selfenforcing alliances without commitment may indeed occur and characterizes both the range of parameter values for which they arise and the nature of transfers in such alliances. Section 5 compares the range of parameters for which positive transfers arise in self-enforcing alliances

\footnotetext{
${ }^{6}$ According to the United States State Department website America.gov Lend-Lease transfers totaled $\$ 50.1$ billion to over 30 different countries. $\$ 31.4$ billion of these funds went to Britain. Although also strong adversaries of the Soviets, Great Britain also transferred aid to the Soviet Union during the conflict. In advocating this aid the strongly anti-Communist Winston Churchill was famously quoted as stating "If Hitler invaded Hell, I would make at least a favourable reference to the Devil in the House of Commons." Soviet distrust of the "imperialist powers" was mutual. Indeed, Stalin refused to ever acknowledge to the Soviet people the scale of the transfers from the United States and Britain.

${ }^{7}$ See for instance, Herring (1973, p.38).
} 
without commitment to the range for which positive individually rational ex ante transfers would arise between players 1 and 2 when complete and binding contingent commitments may be made as to the ex post division of payoffs. We call alliances in which such commitments can be made alliances with complete commitment. Section 6 concludes and outlines extensions.

\section{The Coalitional Colonel Blotto Game}

\section{Players}

There are 3 players, $\{A, 1,2\}$, and two simultaneous Colonel Blotto games, $G_{1}$ and $G_{2}$. Player $A$ competes in both of the Colonel Blotto games, $G_{1}$ and $G_{2}$. Each player $i \in 1,2$ competes in only one Colonel Blotto game, $G_{i}$ (see the schematic in Figure 1). The Colonel Blotto game $G_{i}$ has $n_{i}$ battlefields, and we will assume that $n_{i} \geq 3, i=1,2 .{ }^{8}$ Each battlefield $j \in\left\{1, \ldots, n_{i}\right\}$ in Colonel Blotto game $G_{i}$ has a payoff of $v_{i}>0$. The total value of Colonel Blotto game $G_{i}, n_{i} v_{i}$, is denoted by $\phi_{i} \equiv n_{i} v_{i}$. The force allocated to each battlefield in each Colonel Blotto game must be nonnegative and each player $i \in A \cup\{1,2\}$ has a normalized budget of $X_{i}$, where player $A$ 's normalized budget is $X_{A}=1$. On each battlefield the player that allocates the higher level of force wins that battlefield. In the case that the players allocate the same level of force on a given battlefield, the player that has the higher level of resources in that Colonel Blotto game wins that battlefield. The specification of the tiebreaking rule does not affect the results as long as no player has less than $\frac{2}{n_{i}}$ times the forces of their opponent in Colonel Blotto game $G_{i}, i=1,2$. In the case that this condition does apply this specification of the tie-breaking rule avoids the need to have the stronger player allocate a level of force that is arbitrarily close to, but above, the weaker player's maximal allocation of force. A range of tie-breaking rules yield similar results.

\footnotetext{
${ }^{8}$ Moving from $n_{i}=2$ to $n_{i} \geq 3$ greatly enlarges the space of feasible $n$-variate distribution functions, and the equilibrium strategies examined in this paper require that $n_{i} \geq 3$.
} 
[Insert Figure 1 here]

\section{Alliances}

In the first stage of the game players 1 and 2 choose whether or not to form an alliance. We focus on the case in which it is not possible for players 1 and 2 to a priori commit to a division rule for the alliance's ex post payoff. In this case each alliance member, conditional on the resources that are available, maximizes the payoff from their individual Colonel Blotto game. To emphasize the point that unilateral transfers between allies may take place in the absence of pure or impure public goods, we assume that neither player's payoff depends on the eventual outcome of his ally's game. However, prior to the play of their respective games, alliance members may reallocate resources among themselves subject to the constraint that the resulting allocation of resources is individually rational for each alliance member.

Since there are many game forms that might govern how mutually beneficial transfers might take place, we instead focus on the following simple question: When does there exist a nonzero and feasible ${ }^{9}$ net transfer, $t$, from player 1 to player 2 (negative $t$ corresponds to a positive net transfer from 2 to 1 ) that strictly increases both allies' payoffs when compared to the case in which $t=0$. In examining this question, we assume that following any choice of $t$ the game that follows is one in which player $A$ observes the resulting budget constraints, $X_{1}^{t} \equiv X_{1}-t$ and $X_{2}^{t} \equiv X_{2}+t$, takes them as given, and then responds optimally in allocating $X_{A}$ across $G_{1}$ and $G_{2}$. We label the resulting allocations of $X_{A}$ across $G_{1}$ and $G_{2}$ by $X_{A 1}$ and $X_{A 2}$, respectively. Once the budgets $\left(X_{1}^{t}, X_{2}^{t}, X_{A 1}, X_{A 2}\right)$ are determined, they become common knowledge and the corresponding complete information simultaneous move Colonel Blotto games $G_{1}$ and $G_{2}$ are played. If such Pareto improving transfers between players 1 and 2 exist, it is reasonable to assume that the allies, in this environment of complete information, can implement some such transfer.

\footnotetext{
${ }^{9}$ Feasibility in this context means that the transfer lies in the interval $\left[-X_{2}, X_{1}\right]$.
} 
Naturally, there are many game forms that might govern the implementation of transfers of the one-dimensional resource between the two allies. For instance, in one version of such a game each ally simultaneously decides upon a nonnegative amount to transfer to its ally. Each ally then observes these amounts and then the allies simultaneously decide whether to accept or reject the offer of its ally. It is straightforward to show that when nonzero transfers exist which are Pareto improving, this offer process can implement one such transfer, the transfer in which the ally making the Pareto improving positive net transfer obtains his most preferred positive net transfer. When nonzero transfers between players 1 and 2 exist that are strictly Pareto improving, we refer to the alliance as a self-enforcing alliance without commitment.

Before examining this game in more detail, it is important to note that one immediate result of Roberson's (2006) characterization of equilibrium payoffs in Colonel Blotto games with asymmetric budgets is that, for a given opposition budget constraint, a player's payoff is nondecreasing in his own budget. Hence, if player $A$ 's allocation of his budget over the two games $G_{i}, i=1,2$, cannot be adjusted in response to transfers, as would be the case if $A$ 's allocation of $X_{A}$ across the two games preceded or was simultaneous with the transfer between players 1 and 2, neither player could possibly strictly benefit from a transfer of resources to his ally.

In the analysis that follows, let $\pi_{i}^{t}$ denote the payoff of the Colonel Blotto game $G_{i}$ to player $i=1,2$ if a self-enforcing alliance without commitment is formed with net transfer from 1 to 2 equal to $t$, and $\pi_{i}^{0}$, denote the payoff to player $i=1,2$ from acting in isolation, with no transfer taking place. By definition, a self-enforcing alliance without commitment forms if and only if $\pi_{i}^{t}>\pi_{i}^{0}$ for some $t \neq 0$ for each $i=1,2$. Thus, a self-enforcing alliance without commitment forms if and only if there exists a reallocation of the alliance members' budgets such that each player $i=1,2$ strictly prefers this to competing with his own endowment, given the corresponding optimal responses of $A$ in allocating his resources. 
Before defining the players' strategies, it is useful to motivate the leadership role the alliance takes in determining transfers. As noted above, if player $A$ cannot condition the allocation of his budget on the available budgets of the alliance members, the private good nature of the expenditure of players 1 and 2 insures that no transfers take place between the two players. However, if $A$ has an opportunity to condition his allocation upon the alliance transfers, a positive transfer from one player to the other may induce a sufficient shift in the optimal budget allocations of player $A$ away from the transferring player's Colonel Blotto game to more than compensate the player for making the positive net transfer. That is, the strategic effect may more than compensate for the direct effect of the transfer for one player, while the direct effect more than compensates for the strategic effect for the other.

Why might it be reasonable to assume that $A$ can condition his allocation across fronts on the transfers of the allies? One reason is that it seems plausible to believe that transfers between alliance members are more easily observed than transfers between different Colonel Blotto games by player $A$. After all, alliance members are different players and reaching agreements to transfer material between players may take longer than solving an individual allocation problem and may involve a public announcement. Moreover, as in the case with the Allies fighting Nazi Germany in The Second World War, it may be the case that the two Colonel Blotto games $G_{1}$ and $G_{2}$ represent two distinct geographically separate fronts in a war and country $A$ can transfer resources between these fronts within the confines of the geographical area that it controls. Finally, the notion of the transfer of resources as a commitment seems more reasonable in the context of a Pareto improving transfer across players than as a shifting of resources controlled by a single player. Any attempt to undo such a commitment would require the compliance and coordination of two decision makers, not just the command of one. 


\section{Strategies}

Let $X_{A 1}$ and $X_{A 2}=1-X_{A 1}$ be player $A$ 's resources allocated to the Colonel Blotto games $G_{1}$ and $G_{2}$, respectively, and $X_{i}^{t}$ be player $i$ 's, $i=1,2$, level of resources utilized in $G_{i}$ after a transfer $t$ is implemented. Each distinct pair of games $\left(G_{1}\left(X_{1}^{t}, X_{A 1}\right), G_{2}\left(X_{2}^{t}, X_{A 2}\right)\right)$ represents a distinct final stage subgame of the overall game. In the final stage, it is well known that for a given $i \in\{1,2\}$, if either $\frac{1}{n_{i}} X_{A i}<X_{i}^{t} \leq X_{A i}$ or $\frac{1}{n_{i}} X_{i}^{t}<X_{A i} \leq X_{i}^{t}$ there exists no pure strategy equilibrium in the final stage Colonel Blotto game $G_{i} \cdot{ }^{10}$

For each player $i \in 1,2$ a mixed strategy in $G_{i}$, which we label a distribution of force for player $i$, is an $n_{i}$-variate distribution function $P_{i}: \mathbb{R}_{+}^{n_{i}} \rightarrow[0,1]$ with support contained in the set of player $i$ 's feasible allocations of force, $\mathfrak{X}_{i}=\left\{\mathbf{x} \in \mathbb{R}_{+}^{n_{i}} \mid \sum_{j=1}^{n_{i}} x_{j} \leq X_{i}^{t}\right\}$, and with a set of univariate marginal distribution functions $\left\{F_{i}^{j}\right\}_{j=1}^{n_{i}}$, one univariate marginal distribution function for each battlefield in player $i$ 's Colonel Blotto game $G_{i}$. The $n_{i^{-}}$ tuple of player $i$ 's allocation of force across their $n_{i}$ battlefields is a random $n_{i}$-tuple drawn from the $n_{i}$-variate distribution $P_{i}$ with the set of univariate marginal distribution functions $\left\{F_{i}^{j}\right\}_{j=1}^{n_{i}}$. Player A's mixed strategy, a distribution of force for player $A$, is a set compromised of an $n_{1}$-variate distribution function $P_{A 1}: \mathbb{R}_{+}^{n_{1}} \rightarrow[0,1]$ and an $n_{2}$-variate distribution function $P_{A 2}: \mathbb{R}_{+}^{n_{2}} \rightarrow[0,1]$. Each of the $n_{i}$-variate distributions $P_{A i}$ has support contained in $\mathfrak{X}_{A i}=\left\{\mathbf{x} \in \mathbb{R}_{+}^{n_{i}} \mid \sum_{j=1}^{n_{i}} x_{j} \leq X_{A i}\right\}$ and has a set of univariate marginal distribution functions $\left\{F_{A i}^{j}\right\}_{j=1}^{n_{i}}$, one univariate marginal distribution function for each battlefield in the Colonel Blotto game $G_{i}$. For each Colonel Blotto game $G_{i}$, the $n_{i}$-tuple of player $A$ 's allocation of force across the $n_{i}$ battlefields is a random $n_{i}$-tuple drawn from the $n_{i}$-variate distribution $P_{A i}$ with the set of univariate marginal distribution functions $\left\{F_{A i}^{j}\right\}_{j=1}^{n_{i}}$.

\footnotetext{
${ }^{10}$ In the cases where $\frac{1}{n_{i}} X_{A i} \geq X_{i}^{t}$ or $\frac{1}{n_{i}} X_{i}^{t} \geq X_{A i}$ there, trivially, exists a pure strategy equilibrium in the game $G_{i}$ and the player with the higher level of resources in that game wins all of the battlefields.
} 


\section{Coalitional Colonel Blotto Games}

The Coalitional Colonel Blotto Game, which we label

$$
\Gamma\left\{G_{1}, G_{2}, X_{A}, X_{1}, X_{2}\right\}
$$

is the multistage game in which players 1 and 2 first implement a feasible net transfer of resources between themselves, player $A$ then observes this transfer and allocates his budget $X_{A}(=1)$ across the two Colonel Blotto games $G_{1}$ and $G_{2}$, and then players 1 and 2 individually compete with player $A$ in their respective Colonel Blotto games by simultaneously announcing distributions of forces to their respective battlefields, subject to their respective budget constraints determined in the previous stages. In the games $G_{1}$ and $G_{2}$ each battlefield is won by the player that provides the higher allocation of force to that battlefield (subject to the tie breaking rules discussed above), and each player's payoff equals the expected value of all battlefields won.

\section{The Final Stage Colonel Blotto Games}

We start our analysis with the final stage subgames $G_{i}, i=1,2$, and work our way back through the game tree. Theorem 1 provides Roberson's (2006) characterization of the unique equilibrium payoffs in the Colonel Blotto game. For further details on the nature of the equilibrium strategies see Roberson (2006). To simplify the exposition we adopt the following notation: let $\bar{X}_{i}=\max \left\{X_{A i}, X_{i}^{t}\right\}$ and $\underline{X}_{i}=\min \left\{X_{A i}, X_{i}^{t}\right\}$ for $i=1,2$. Let the player with $\underline{X}_{i}$ forces be denoted as player $k$, and the player with $\bar{X}_{i}$ forces be denoted as player $-k$.

Theorem 1 (Roberson (2006)). The unique Nash equilibrium payoffs of the final stage Colonel Blotto game $G_{i}$ in the game $\Gamma\left\{G_{1}, G_{2}, X_{A}, X_{1}, X_{2}\right\}$ are given as follows:

A. If $\underline{X}_{i}$ and $\bar{X}_{i}$ satisfy $\frac{2}{n_{i}} \leq \frac{\underline{X}_{i}}{\bar{X}_{i}} \leq 1$, then the payoff for player $k$ is $\phi_{i}\left(\frac{\underline{X}_{i}}{2 \bar{X}_{i}}\right)$ and the payoff 
for player $-k$ is $\phi_{i}\left(1-\frac{\underline{X}_{i}}{2 \bar{X}_{i}}\right)$.

B. If $\underline{X}_{i}$ and $\bar{X}_{i}$ satisfy $\frac{1}{n_{i}-1} \leq \frac{\underline{X}_{i}}{\bar{X}_{i}}<\frac{2}{n_{i}}$, then the payoff for player $k$ is $\phi_{i}\left(\frac{2}{n_{i}}-\frac{2 \bar{X}_{i}}{n_{i}^{2} \underline{X}_{i}}\right)$ and the payoff for player $-k$ is $\phi_{i}\left(1-\frac{2}{n_{i}}+\frac{2 \bar{X}_{i}}{n_{i}^{2} \underline{X}_{i}}\right)$.

C. If $\underline{X}_{i}$ and $\bar{X}_{i}$ satisfy $\frac{1}{n_{i}}<\frac{\underline{X}_{i}}{\bar{X}_{i}}<\frac{1}{n_{i}-1}$, then define $m=\left\lceil\frac{\underline{X}_{i}}{\bar{X}_{i}-\underline{X}_{i}\left(n_{i}-1\right)}\right\rceil$, and note that $2 \leq$ $m<\infty$. The payoff for player $k$ is $\phi_{i}\left(\frac{2 m-2}{m n_{i}^{2}}\right)$, and the payoff for player $-k$ is $\phi_{i}\left(1-\frac{2 m-2}{m n_{i}^{2}}\right)$.

For a proof of Theorem 1 see Roberson (2006). This proof establishes the existence of equilibrium $n_{i}$-variate distributions which are feasible (i.e., with supports contained in $\left\{\mathbf{x} \in \mathbb{R}_{+}^{n_{i}} \mid \sum_{j=1}^{n_{i}} x_{j}=\underline{X}_{i}\right\}$ and $\left\{\mathbf{x} \in \mathbb{R}_{+}^{n_{i}} \mid \sum_{j=1}^{n_{i}} x_{j}=\bar{X}_{i}\right\}$ respectively) and that provide the equilibrium payoffs given above. Note that the final stage games $G_{1}$ and $G_{2}$ are constant-sum games, guaranteeing the uniqueness of the equilibrium payoffs. These payoffs are illustrated in Figure 2 as a function of $\frac{\underline{X}_{i}}{\bar{X}_{i}}$. A salient feature of this characterization is that as the number of battlefields, $n_{i}$, becomes large, the ranges of $\frac{\underline{X}_{i}}{\bar{X}_{i}}$ covered by parts (B) and (C) of Theorem 1 collapse to zero, and the weaker player's payoff (in these ranges) goes to zero as well. We use these facts in the analysis of the second stage game that follows.

[Insert Figure 2 here]

\section{Stages One and Two: Alliances and Resource Allo- cations}

We begin in stage two with player $A$ 's optimal allocation of resources between the two Colonel Blotto games. The primitives in this section are the payoffs derived in the previous section. Given the above characterization, it follows that the form of player $A$ 's payoff function depends critically on the transfer of resources between players 1 and 2 in the first period. In fact for player $A$ there are 64 different regions each with a distinct form for the payoff function. These regions correspond to the cases where either $\frac{X_{i}^{t}}{X_{A i}}$ or $\frac{X_{A i}}{X_{i}^{t}}, i=1,2$, 
satisfy one of the three conditions of Theorem 1, or one player has more than $n_{i}$ times the budget of the other in $G_{i}$.

For example, assume that $X_{A}=1>X_{1}^{t}+X_{2}^{t}$. If player $A$ divides his resources between the two Colonel Blotto games such that $\frac{2}{n_{1}} \leq \frac{X_{1}^{t}}{X_{A 1}} \leq 1$ and $\frac{2}{n_{2}} \leq \frac{X_{2}^{t}}{X_{A 2}} \leq 1$ then player $A$ 's payoff function is:

$$
\pi_{A}\left(\left\{X_{A i}, X_{i}^{t}\right\}_{i=1,2}\right)=\phi_{1}\left(1-\frac{X_{1}^{t}}{2 X_{A 1}}\right)+\phi_{2}\left(1-\frac{X_{2}^{t}}{2 X_{A 2}}\right) .
$$

The payoff functions for the remaining regions are similarly constructed.

To simplify the analysis the number of battlefields $n_{i}$ is assumed to be arbitrarily large. (However, the total value of each Colonel Blotto game $\phi_{i}=n_{i} v_{i}$ is held constant.) Thus, the number of different regions collapses from 64 to 4 , which are given by $\frac{2}{n_{i}} \leq \frac{X_{i}^{t}}{X_{A i}} \leq 1$ and $\frac{2}{n_{i}} \leq \frac{X_{A i}}{X_{i}^{t}} \leq 1$ for each Colonel Blotto game $G_{i}, i=1,2$. For given post-transfer levels of resources of players 1 and $2, X_{1}^{t}$ and $X_{2}^{t}$ respectively, player $A$ 's payoffs in each Colonel Blotto game are shown in Figure 3 below.

[Insert Figure 3 here]

Player $A$ 's optimal second stage allocation of resources between the two Colonel Blotto games is determined by the marginal payoffs in each Colonel Blotto game. In particular, there are four qualitatively distinct cases of optimal resource allocations for player $A$. These correspond to the four distinct regions of $\left(X_{i}^{t}, X_{-i}^{t}\right)$ pairs illustrated in Figure 4.

Case 1. Suppose $\frac{\phi_{i}}{\phi_{-i}}>\frac{\max \left\{\left(X_{i}^{t}\right)^{2}, 1\right\}}{X_{i}^{t} X_{-i}^{t}}$ or $1>X_{i}^{t}$ and $\frac{\phi_{i}}{\phi_{-i}}=\frac{1}{X_{i}^{t} X_{-i}^{t}}$. Then player $A$ allocates all of his resources to Colonel Blotto game $G_{i}$.

In Case 1, each unit of resource that player $A$ allocates to the Colonel Blotto game $G_{i}$ has a marginal payoff that is higher than the first unit allocated to $G_{-i}$. If the initial endowments 
$X_{1}^{0}, X_{2}^{0}$ are such that this case holds, it is clear that there can be no nonzero net transfer that strictly improves upon the allocation for both players, since player $-i$ cannot do strictly better.

Case 2. Suppose $\frac{\phi_{i}}{\phi_{-i}}>\frac{X_{i}^{t}}{X_{-i}^{t}}$ and $0<1-\left(\frac{\phi_{i} X_{i}^{t} X_{-i}^{t}}{\phi_{-i}}\right)^{\frac{1}{2}} \leq X_{-i}^{t}$. Then player $A$ allocates $X_{A i}=\left(\frac{\phi_{i} X_{i}^{t} X_{-i}^{t}}{\phi_{-i}}\right)^{\frac{1}{2}}$ to Colonel Blotto game $G_{i}$ and $X_{A(-i)}=1-\left(\frac{\phi_{i} X_{i}^{t} X_{-i}^{t}}{\phi_{-i}}\right)^{\frac{1}{2}}$ to Colonel Blotto game $G_{-i}$.

In Case 2, A's budget is sufficiently large that it is optimal to allocate a level of resources greater than $X_{i}^{t}$ to the Blotto game $G_{i}, X_{A i}>X_{i}^{t}$, thereby hitting the range of diminishing returns (see Figure 3). At the margin A equates the return to an extra unit of resource allocated to game $G_{i}$ to the constant marginal return that he receives for allocating $X_{A(-i)}<$ $X_{-i}^{t}$. That is $\frac{\phi_{-i}}{2 X_{-i}^{t}}=\frac{\phi_{i} X_{i}^{t}}{2\left(X_{A i}\right)^{2}}$ (see Figure 3), yielding $X_{A i}=\left(\frac{\phi_{i} X_{i}^{t} X_{-i}^{t}}{\phi_{-i}}\right)^{\frac{1}{2}}$. Player $A^{\prime}$ 's remaining forces $0<1-\left(\frac{\phi_{i} X_{i}^{t} X_{-i}^{t}}{\phi_{-i}}\right)^{\frac{1}{2}}<X_{-i}^{t}$ are allocated to the remaining Colonel Blotto game, $G_{-i}$.

Case 3. Suppose $\frac{\phi_{i}}{\phi_{-i}} \geq \frac{X_{i}^{t}}{X_{-i}^{t}}$ and $1-\left(\frac{\phi_{i} X_{i}^{t} X_{-i}^{t}}{\phi_{-i}}\right)^{\frac{1}{2}}>X_{-i}^{t}$. Then player $A$ allocates $X_{A i}=\frac{\left(\phi_{i} X_{i}^{t}\right)^{\frac{1}{2}}}{\left(\phi_{i} X_{i}^{t}\right)^{\frac{1}{2}}+\left(\phi_{-i} X_{-i}^{t}\right)^{\frac{1}{2}}}$ to Colonel Blotto game $G_{i}$ and $X_{A(-i)}=\frac{\left(\phi_{-i} X_{-i}^{t}\right)^{\frac{1}{2}}}{\left(\phi_{i} X_{i}^{t}\right)^{\frac{1}{2}}+\left(\phi_{-i} X_{-i}^{t}\right)^{\frac{1}{2}}}$ to Colonel Blotto game $G_{-i}$.

In Case 3 player $A$ has a sufficient level of resources to be able to set the marginal payoffs from the two Colonel Blotto games equal at levels greater than the corresponding resource levels of players 1 and 2 . In particular, player $A$ chooses $X_{A i}$ and $X_{A(-i)}$ such that $\frac{\phi_{-i} X_{-i}^{t}}{2\left(X_{A(-i)}\right)^{2}}=\frac{\phi_{i} X_{i}^{t}}{2\left(X_{A i}\right)^{2}}$ (see Figure 3).

Case 4. Suppose $\frac{\phi_{i}}{\phi_{-i}}=\frac{X_{i}^{t}}{X_{-i}^{t}}$ and $1 \leq X_{1}^{0}+X_{2}^{0}$. Then any pair $\left(X_{A 1}, X_{A 2}\right)$ such that $X_{A 1}+$ $X_{A 2}=1$ and $X_{A i} \leq X_{i}^{t}, i=1,2$ is an optimal response of player $A$.

In Case 4 any allocation by player $A$ in which $X_{A i} \leq X_{i}^{t}, i=1,2$ sets the marginal payoffs from the two Colonel Blotto Games equal. As is shown in Section 5, if players 1 and 
2 had the ability to commit to binding agreements and $1 \leq X_{1}^{0}+X_{2}^{0}$, then the transfer, $t$, which sets $\frac{\phi_{i}}{\phi_{-i}}=\frac{X_{i}^{t}}{X_{-i}^{t}}$ maximizes the sum of the two players' payoffs. Thus, it is clear that in Case 4 there can be no nonzero net transfer that strictly improves upon the allocation for both players.

Figure 4 illustrates the ranges of $\left(X_{i}^{t}, X_{-i}^{t}\right)$ pairs corresponding to the cases described above for values of $\phi_{i}$ and $\phi_{-i}$ such that $\frac{\phi_{i}}{\phi_{-i}} \geq 1$. The analysis is analogous when $\frac{\phi_{i}}{\phi_{-i}}<1$.

\section{[Insert Figure 4 here]}

We now determine when there exists a nonzero transfer $t$ from player 1 to player 2 that strictly Pareto improves upon their initial endowments of the resource. The primitives at this stage are the $\left(X_{1}^{t}, X_{2}^{t}\right)$-contingent subgame payoffs arising when player $A$ optimally responds as detailed in Cases 1 through 4 above, and the resulting Colonel Blotto game payoffs are given as in Theorem 1, part (A).

By definition a self-enforcing alliance without commitment exists if and only if there exists a $t \neq 0$ such that

$$
\pi_{i}^{t}\left(X_{i}^{t}, X_{A i}\left(X_{1}^{t}, X_{2}^{t}\right)\right)>\pi_{i}^{0}\left(X_{i}^{0}, X_{A i}\left(X_{1}^{0}, X_{2}^{0}\right)\right)
$$

in each of the respective games $G_{i}, i=1,2$.

Clearly if the initial resource endowments $\left(X_{1}^{0}, X_{2}^{0}\right)$ satisfy the conditions of Case 1 , then there is no incentive for a non-zero transfer to take place (player $-i$ is already receiving his highest feasible payoff). ${ }^{11}$ The following two propositions examine alliance formation when the initial resource endowments satisfy the conditions of Cases 2 and 3, respectively.

\footnotetext{
${ }^{11}$ While our focus is on alliance transfers that strictly benefit both alliance members, it is instructive to note that in the portion of the Case 1 region in which $1 \leq X_{i}^{0}$ or $1>X_{i}^{0}$ and $\frac{\phi_{i}}{\phi_{-i}} \neq \frac{1}{X_{i}^{0} X_{-i}^{0}}$, player $-i$ is indifferent between keeping his endowment and making a transfer to player $i$ that leaves the endowment pair within the region, whereas player $i$ prefers to accept any such transfer.
} 
Proposition 1. Suppose $\left(X_{1}^{0}, X_{2}^{0}\right)$ satisfies the conditions of Case 2. Then a self-enforcing alliance without commitment exists in which player -i transfers a net positive level of resources to player $i$ if and only if

$$
X_{i}^{0}+X_{-i}^{0}>2\left(\frac{\phi_{-i} X_{i}^{0}}{\phi_{i} X_{-i}^{0}}\right)^{\frac{1}{2}}
$$

No self-enforcing alliance exists in which player $i$ transfers a positive net level of resources.

Proof. With the initial endowments satisfying the conditions of Case 2, player A's optimal allocation of forces between the two Blotto games is determined by $\frac{\phi_{-i}}{2 X_{-i}^{0}}=\frac{\phi_{i} X_{i}^{0}}{2\left(X_{A i}\right)^{2}}$, and thus $X_{A i}=\left(\frac{\phi_{i} X_{1}^{0} X_{2}^{0}}{\phi_{-i}}\right)^{\frac{1}{2}}$ and $X_{A(-i)}=1-\left(\frac{\phi_{i} X_{1}^{0} X_{2}^{0}}{\phi_{-i}}\right)^{\frac{1}{2}}$. Given player $A$ 's optimal stage 2 allocation of resources between the two Colonel Blotto games with no transfers, player $i$ 's payoff is

$$
\pi_{i}^{0}\left(X_{i}^{0}, X_{A i}\left(X_{1}^{0}, X_{2}^{0}\right)\right)=\frac{1}{2}\left(\frac{\phi_{i} \phi_{-i} X_{i}^{0}}{X_{-i}^{0}}\right)^{\frac{1}{2}}
$$

and player $-i$ 's payoff is

$$
\pi_{-i}^{0}\left(X_{-i}^{0}, X_{A(-i)}\left(X_{1}^{0}, X_{2}^{0}\right)\right)=\phi_{-i}-\frac{\phi_{-i}}{2 X_{-i}^{0}}+\frac{1}{2}\left(\frac{\phi_{i} \phi_{-i} X_{i}^{0}}{X_{-i}^{0}}\right)^{\frac{1}{2}}
$$

Note that any positive net transfers $\tau$ from $i$ to $-i$ would result in the pair $\left(X_{1}^{t}, X_{2}^{t}\right)$ satisfying the conditions of Case $2\left(\frac{\phi_{i}}{2 X_{i}^{0}-2 \tau}\right.$ increases while $\frac{\phi_{-i}}{2 X_{-i}^{0}+2 \tau}$ decreases $)$, and that in this case player $A$ 's optimal allocation of forces between the two Blotto games is determined by $\frac{\phi_{-i}}{2 X_{-i}^{0}+2 \tau}=\frac{\phi_{i}\left(X_{i}^{0}-\tau\right)}{2\left(X_{A i}\right)^{2}}$. Thus player $i$ 's payoff from such a transfer is

$$
\pi_{i}^{\tau}\left(X_{i}^{0}-\tau, X_{A i}\left(X_{i}^{0}-\tau, X_{-i}^{0}+\tau\right)\right)=\frac{1}{2}\left(\frac{\phi_{i} \phi_{-i}\left(X_{i}^{0}-\tau\right)}{\left(X_{-i}^{0}+\tau\right)}\right)^{\frac{1}{2}} .
$$

It follows immediately that since $\frac{\partial \pi_{i}^{\tau}}{\partial \tau}<0$ for all feasible positive net transfers $\tau$, it is clear 
that any nonzero strictly Pareto improving transfer must be from player $-i$ to player $i$. Furthermore, it is also clear that any such transfer of resources from player $-i$ could not result in the pair $\left(X_{1}^{t}, X_{2}^{t}\right)$ satisfying the conditions of Case 3 or Case 2 with $\frac{\phi_{-i}}{X_{-i}^{0}-\tau}>\frac{\phi_{i}}{X_{i}^{0}+\tau}$ (i.e. the roles reversed) since in both cases player $-i$ would be worse off. Thus we can restrict our attention to alliance transfers from player $-i$ to player $i$ in which the resulting levels of resources remain in the current Case 2.

If a positive net transfer, $\tau$, of resources from player $-i$ to player $i$ takes place, resulting in an allocation that remains in Case 2, player $A$ 's optimal allocation of forces between the two Blotto games is determined by the marginal condition $\frac{\phi_{-i}}{2 X_{-i}^{0}-2 \tau}=\frac{\phi_{i}\left(X_{i}^{0}+\tau\right)}{2\left(X_{A i}\right)^{2}}$ and thus player $i$ 's payoff is

$$
\pi_{i}^{\tau}\left(X_{i}^{0}+\tau, X_{A i}\left(X_{i}^{0}+\tau, X_{-i}^{0}-\tau\right)\right)=\frac{1}{2}\left(\frac{\phi_{i} \phi_{-i}\left(X_{i}^{0}+\tau\right)}{\left(X_{-i}^{0}-\tau\right)}\right)^{\frac{1}{2}}
$$

and player $-i$ 's payoff is

$$
\begin{gathered}
\pi_{-i}^{\tau}\left(X_{-i}^{0}-\tau, X_{A(-i)}\left(X_{i}^{0}+\tau, X_{-i}^{0}-\tau\right)\right)= \\
\phi_{-i}-\frac{\phi_{-i}}{2 X_{-i}^{0}-2 \tau}+\frac{1}{2}\left(\frac{\phi_{i} \phi_{-i}\left(X_{i}^{0}+\tau\right)}{\left(X_{-i}^{0}-\tau\right)}\right)^{\frac{1}{2}} .
\end{gathered}
$$

Note that $\frac{\partial \pi_{i}^{\tau}}{\partial \tau}=\frac{\left(\phi_{i} \phi_{-i}\right)^{1 / 2}\left(X_{i}^{0}+X_{-i}^{0}\right)}{4\left(X_{-i}^{0}-\tau\right)^{3 / 2}\left(X_{i}^{0}+\tau\right)^{1 / 2}}$, which is positive for all $\tau$. Thus player $i$ is always willing to accept a transfer $\tau>0$. It is straightforward to show that,

$$
\frac{\partial \pi_{-i}^{\tau}}{\partial \tau}=-\frac{\phi_{-i}}{2\left(X_{-i}^{0}-\tau\right)^{2}}+\frac{\left(\phi_{i} \phi_{-i}\right)^{1 / 2}\left(X_{i}^{0}+X_{-i}^{0}\right)}{4\left(X_{-i}^{0}-\tau\right)^{3 / 2}\left(X_{i}^{0}+\tau\right)^{1 / 2}}
$$

Clearly, if $\left.\frac{\partial \pi_{-i}^{\tau}}{\partial \tau}\right|_{\tau=0}>0$, a sufficiently small positive transfer would benefit $-i$ as well. Moreover, it is straightforward to show that if $\left.\frac{\partial \pi_{-i}^{\tau}}{\partial \tau}\right|_{\tau=0} \leq 0$ then $\frac{\partial \pi_{-i}^{\tau}}{\partial \tau}$ will remain nonpositive for all $\tau>0$ such that $\tau<X_{-i}^{0}$. Hence player $-i$ will strictly benefit from a positive transfer to 
player $i$ if and only if $\left.\frac{\partial \pi_{-i}^{\tau}}{\partial \tau}\right|_{\tau=0}>0$. This holds if and only if $X_{i}^{0}+X_{-i}^{0}>2\left(\frac{\phi_{-i} X_{i}^{0}}{\phi_{i} X_{-i}^{0}}\right)^{\frac{1}{2}}$. Q.E.D.

Proposition 2. Suppose $\left(X_{1}^{0}, X_{2}^{0}\right)$ satisfies the conditions of Case 3. Then a self-enforcing alliance without commitment exists in which player $-i$ transfers a net positive level of resources to player $i$ if and only if

$$
1-\frac{X_{i}^{0}}{X_{-i}^{0}}>2\left(\frac{\phi_{-i} X_{i}^{0}}{\phi_{i} X_{-i}^{0}}\right)^{\frac{1}{2}}
$$

No self-enforcing alliance exists in which player $i$ transfers a positive net level of resources.

Proof. With the initial endowments satisfying the conditions of Case 3, player $A$ 's optimal allocation of forces between the two Blotto games is determined by $\frac{\phi_{i} X_{i}^{0}}{2\left(X_{A i}\right)^{2}}=\frac{\phi_{-i} X_{-i}^{0}}{2\left(X_{A(-i)}\right)^{2}}$, and thus $X_{A 1}=\frac{\left(\phi_{1} X_{1}^{0}\right)^{\frac{1}{2}}}{\left(\phi_{1} X_{1}^{0}\right)^{\frac{1}{2}}+\left(\phi_{2} X_{2}^{0}\right)^{\frac{1}{2}}}$ and $X_{A 2}=\frac{\left(\phi_{2} X_{2}^{0}\right)^{\frac{1}{2}}}{\left(\phi_{1} X_{1}^{0}\right)^{\frac{1}{2}}+\left(\phi_{2} X_{2}^{0}\right)^{\frac{1}{2}}}$. Given player $A$ 's optimal stage 2 allocation of forces between the two Colonel Blotto games player $i$ 's, $i=1$, 2, payoff with a zero transfer is

$$
\pi_{i}^{0}\left(X_{i}^{0}, X_{A i}\left(X_{1}^{0}, X_{2}^{0}\right)\right)=\frac{\phi_{i}}{2}\left(X_{i}^{0}+\left(\frac{\phi_{-i} X_{i}^{0} X_{-i}^{0}}{\phi_{i}}\right)^{\frac{1}{2}}\right)
$$

If a positive net transfer, $\tau>0$, of resources from player $-i$ to player $i$ takes place, it is feasible that the resulting allocation may remain in Case 3 or may switch to Case 2.

First looking at transfers within Case 3, player $A$ 's optimal allocation of resources between the two Blotto games is determined by the marginal condition $\frac{\phi_{i}\left(X_{i}^{0}-\tau\right)}{2\left(X_{A i}\right)^{2}}=\frac{\phi_{-i}\left(X_{-i}^{0}+\tau\right)}{2\left(X_{A(-i)}\right)^{2}}$. Hence, player $-i$ 's payoff is given by

$$
\begin{gathered}
\pi_{-i}^{\tau}\left(X_{-i}^{0}-\tau, X_{A(-i)}\left(X_{i}^{0}+\tau, X_{-i}^{0}-\tau\right)\right)= \\
\frac{\phi_{-i}}{2}\left(X_{-i}^{0}-\tau+\left(\frac{\phi_{i}\left(X_{-i}^{0}-\tau\right)\left(X_{i}^{0}+\tau\right)}{\phi_{-i}}\right)^{\frac{1}{2}}\right)
\end{gathered}
$$


and player $i$ 's payoff follows directly. Clearly if $\left.\frac{\partial \pi_{-i}^{\tau}}{\partial \tau}\right|_{\tau=0}>0$ a sufficiently small positive transfer would benefit $-i$, and if $\left.\frac{\partial \pi_{i}^{\tau}}{\partial \tau}\right|_{\tau=0}>0$ a sufficiently small transfer would benefit $i$. Moreover, it is straightforward to show that if $\left.\frac{\partial \pi_{-i}^{\tau}}{\partial \tau}\right|_{\tau=0} \leq 0$ then $\frac{\partial \pi_{-i}^{\tau}}{\partial \tau}$ will remain nonpositive for all $\tau>0$ such that $\tau<X_{-i}^{0}$. In addition, for all $\tau, \frac{\partial \pi_{i}^{\tau}}{\partial \tau}>\frac{\partial \pi_{-i}^{\tau}}{\partial \tau}$. Hence within the range of transfers that remain in Case 3, both players 1 and 2 will strictly benefit from a net positive transfer from player $-i$ to player $i$ if and only if $\left.\frac{\partial \pi_{-i}^{\tau}}{\partial \tau}\right|_{\tau=0}>0$.

It is straightforward to show that

$$
\frac{\partial \pi_{-i}^{\tau}}{\partial \tau}=\frac{\phi_{-i}}{2}\left[-1+\frac{1}{2}\left(\frac{\phi_{i}}{\phi_{-i}}\right)^{\frac{1}{2}} \frac{X_{-i}^{0}-X_{i}^{0}-2 \tau}{\left(\left(X_{-i}^{0}-\tau\right)\left(X_{i}^{0}+\tau\right)\right)^{\frac{1}{2}}}\right]
$$

Thus, there exists a strictly Pareto improving transfer $\tau>0$, from $-i$ to $i$, that remains in the range of allocations covered by Case 3 if and only if $\frac{X_{-i}^{0}-X_{i}^{0}}{\left(X_{i}^{0} X_{-i}^{0}\right)^{\frac{1}{2}}}>2\left(\frac{\phi_{-i}}{\phi_{i}}\right)^{\frac{1}{2}}$. We claim that this is also a necessary condition for the existence of a strictly Pareto improving transfer from $-i$ to $i$ that switches to Case 2. This results from the fact that the subset of Case 2 allocations where $\left.\frac{\partial \pi_{-i}^{\tau}}{\partial \tau}\right|_{\tau=0}>0$ (delineated in Proposition 1) may be reached through a transfer from $-i$ to $i$ only if the initial Case 3 allocation satisfies the condition of Proposition 2.

A similar condition holds for player $i$. In examining transfers $\tau>0$ from $i$ to $-i$, $\left.\frac{\partial \pi_{i}^{\tau}}{\partial \tau}\right|_{\tau=0}>0$ is equivalent to $\frac{X_{i}^{0}-X_{-i}^{0}}{\left(X_{i}^{0} X_{-i}^{0}\right)^{\frac{1}{2}}}>2\left(\frac{\phi_{i}}{\phi_{-i}}\right)^{\frac{1}{2}}$. However, no initial endowment in which $\frac{\phi_{i}}{\phi_{-i}} \geq \frac{X_{i}^{0}}{X_{-i}^{0}}$ satisfies this constraint. Thus, player $i$ never offers a positive net transfer to player $-i$ that results in an allocation in Case 3. As shown in Proposition 1, once in Case 2 player $i$ also never offers a positive net transfer to player $-i$. It follows directly that given an initial endowment in Case 3 there exists no strictly Pareto improving positive net transfer from player $i$ to player $-i$ that crosses over into Case 2. Thus, player $i$ never offers a positive net transfer to player $-i$. Q.E.D. 
Propositions 1 and 2 demonstrate that there are several ranges of parameters in which endogenous unilateral transfers take place. That is, a self-enforcing alliance without commitment forms. The set of $\left(X_{i}^{0}, X_{-i}^{0}\right)$ pairs for which such an alliance forms is illustrated in Figure 5 for the case in which $X_{A}=1$. The $\left(X_{i}^{0}, X_{-i}^{0}\right)$ pairs satisfying the conditions of Cases 2 or 3 and lying in the region above and to the left of the bold lines are the initial endowments for which these alliances arise.

[Insert Figure 5 here]

As is evident from Figure 5 and the inequalities that determine this region in the statements of the two propositions, self-enforcing alliances without commitment form only when players 1 and 2 have sufficiently asymmetric endowments both in absolute terms and relative to the corresponding values of their Blotto games. In particular, in the region of endowments corresponding to Case 3, the boundary delineating the set of endowments for which these alliances form is linear (see Figure 5). Throughout this region, self-enforcing alliances without commitment form if and only if the ratio of the initial endowments $\frac{X_{-i}^{0}}{X_{i}^{0}}$ exceeds a constant threshold, which is greater than $\max \left\{1, \frac{\phi_{-i}}{\phi_{i}}\right\}$. This insures that alliance transfers only flow from player $-i$, the player with the higher endowment, to player $i$, the player with the lower endowment and only if the ratio of their endowments $\frac{X_{-i}^{0}}{X_{i}^{0}}$ exceeds the ratio of Blotto game values $\frac{\phi_{-i}}{\phi_{i}}$.

In the region of endowments corresponding to Case 2, the boundary of the set of endowments for which these alliances form is concave in $X_{i}^{0}$. Within this region, as the sum of the endowments, $X_{i}^{0}+X_{-i}^{0}$, increases, the threshold value of $\frac{X_{-i}^{0}}{X_{i}^{0}}$ above which alliances form decreases. When $\frac{\phi_{-i}}{\phi_{i}}<1$, as in panel (a) of Figure 5 , the boundary of the set of endowments for which these alliances form intersects the boundary of the region corresponding to Case 1 along the $45^{\circ}$ line. One consequence (as is illustrated in Figure 5, panel (a)) is that there exist 
parameter configurations for which self-enforcing alliances without commitment arise even though the initial endowments are arbitrarily close to equality. When $\frac{\phi_{-i}}{\phi_{i}}>1$, as in panel (b) of Figure 5, the boundary of this set intersects the line $X_{-i}^{t}=\frac{\phi_{-i}}{\phi_{i}} X_{i}^{t}$ before it reaches the boundary of the Case 1 region. Indeed, from the condition provided in Proposition 2, this happens precisely when $X_{i}^{0}+X_{-i}^{0}=2$. One consequence, (as is illustrated in Figure 5, panel (b)) is that there exist parameter configurations for which self-enforcing alliances without commitment arise even though the ratio of the initial endowments $\frac{X_{-i}^{0}}{X_{i}^{0}}$ is arbitrarily close to the ratio of Blotto game values $\frac{\phi_{-i}}{\phi_{i}} \cdot{ }^{12}$ Finally, as in the region of endowments corresponding to Case 3, for Case 2 endowments alliance transfers always flow from player $-i$, the player with the higher endowment, to player $i$, the player with the lower endowment, and only if the ratio of their endowments $\frac{X_{-i}^{0}}{X_{i}^{0}}$ exceeds the ratio of Blotto game values $\frac{\phi_{-i}}{\phi_{i}}$.

In this sense, the nature of transfers in our model conform to a version of the "exploitation hypothesis". When self-enforcing alliances without commitment form, transfers flow from the player who is resource rich to the player who is resource poor, both in absolute terms and relative to the total value at stake in their respective Colonel Blotto games with player A.

Moreover, when self-enforcing alliances without commitment form, it must be the case that the combination of direct and strategic effects of the unilateral transfer benefits both allies. Clearly, since the direct effect harms the player making the transfer and benefits the player receiving the transfer, it must be the case that the strategic effect benefits the transferring player and harms the receiving player (if player $A$ moves resources away from the game of the transferring player, these resources flow to the game with the receiving player). In this context, it is interesting to identify the source of a breakdown of the existence of self-enforcing alliances without commitment, that is, whether it is the relatively resource

\footnotetext{
${ }^{12}$ This holds for initial endowments which are (1) above the line $X_{-i}^{t}=\frac{\phi_{-i}}{\phi_{i}} X_{i}^{t}$ and (2) satisfy $2 \leq$ $X_{-i}^{0}+X_{i}^{0} \leq 1+\frac{\phi_{-i}}{\phi_{i}}$.
} 
poor ally who declines to receive a transfer or the relatively rich ally who declines to initiate the transfer. The details of the proofs of these two Propositions indicate that it is always the resource rich ally whose incentive constraint binds first. That is, the region where such alliances form is bounded by the willingness of the player making the transfer.

These results can also be interpreted in light of Dresher's "no soft-spot" principle which implies that the alliance should transfer resources from the harder front to the softer front up until the point that each front is equally attractive to the common opponent. In the context of our multi-front Colonel Blotto game, the relevant measure of attractiveness or softness - from the point of view of the common opponent — is the ratio of the value of a front to the alliance's post-transfer level of force on the front (henceforth, a front's ratio of value to alliance force). In any self-enforcing alliance without commitment, the alliance's equilibrium transfers flow from the harder front towards the softer front, thereby making the soft spot less soft. In the next section we examine alliances with complete commitment. In this case we explicitly solve for the alliance's equilibrium transfer of resources and find that these transfers result in no soft spots, or equivalently each front has the same ratio of value to alliance force.

\section{Alliances with Complete Commitment}

As a benchmark for the analysis of self-enforcing alliances without commitment, it is useful to examine the nature of ex ante transfers that would arise between players 1 and 2 if complete and binding commitments could be made concerning the ex post division of payoffs. We call alliances in which such commitment can be made alliances with complete commitment.

In the presence of complete and binding commitments an optimal ex ante transfer solves

$$
\max _{t} \pi_{1}^{t}\left(X_{1}^{t}, X_{A 1}\left(X_{1}^{t}, X_{2}^{t}\right)\right)+\pi_{2}^{t}\left(X_{2}^{t}, X_{A 2}\left(X_{1}^{t}, X_{2}^{t}\right)\right)
$$


Note that although Propositions 1 and 2 identify the parameter ranges in which there exist Pareto-improving alliance transfers, this optimization problem identifies the Pareto optimal transfers for the alliance. In Proposition 3 we show that an optimal transfer leads to the no soft-spot outcome in which $X_{-i}^{t}=\frac{\phi_{-i}}{\phi_{i}} X_{i}^{t}$. Hence, unless the initial endowments satisfy $X_{-i}^{0}=\frac{\phi_{-i}}{\phi_{i}} X_{i}^{0}$ nonzero transfers of resource endowments will always take place. ${ }^{13}$

Proposition 3. Let $\hat{X}=X_{1}^{0}+X_{2}^{0}$. In any alliance with complete commitment, the allocation of the alliance budget to the two Colonel Blotto games is $X_{i}^{t}=\frac{\hat{X} \phi_{i}}{\phi_{i}+\phi_{-i}}$ and $X_{-i}^{t}=\frac{\hat{X} \phi_{-i}}{\phi_{i}+\phi_{-i}}$. Thus, the alliance transfers result in $\frac{\phi_{i}}{\phi_{-i}}=\frac{X_{i}^{t}}{X_{-i}^{t}}$. If $X_{-i}^{0}=\frac{\phi_{-i}}{\phi_{i}} X_{i}^{0}$, then no transfers take place.

Proof. We begin with the case that $\hat{X} \geq 1$. Thus, in the alliance with complete commitment, the allocation of the alliance budget to the two Colonel Blotto games may satisfy the conditions for Case 1, Case 2, or Case 4 (see Figure 4). Clearly, any allocation by the alliance that satisfies the conditions for Case 1 is not an equilibrium strategy. In Case 1 the alliance wins all of the battlefields in Blotto game $-i$ and player $A$ allocates zero resources to Blotto game $-i$. Thus, the alliance can strictly increase its payoff by diverting resources from the Blotto game $-i$ to the Blotto game $i$ up until the point at which $\frac{\phi_{i}}{\phi_{-i}}=\frac{X_{i}^{t}}{X_{-i}^{t}}$, as in Case 4 , or $0<1-\left(\frac{\phi_{i} X_{i}^{t} X_{-i}^{t}}{\phi_{-i}}\right)^{\frac{1}{2}}$, as in Case 2 .

Similarly, any allocation by the alliance that satisfies the conditions for Case 2 is not an equilibrium strategy. In particular note that in Case 2 the joint payoff of the alliance, $\pi_{12}^{\tau} \equiv \pi_{1}^{\tau}+\pi_{2}^{\tau}$, given any allocation, $\left(X_{i}^{0}, X_{-i}^{0}\right)$ in Case 2 and any transfer $\tau>0$ such that $\frac{X_{i}^{0}+\tau}{\phi_{i}}<\frac{X_{-i}^{0}-\tau}{\phi_{-i}}$, is given by

$$
\begin{gathered}
\pi_{12}^{\tau}\left(X_{i}^{0}+\tau, X_{-i}^{0}-\tau\right)= \\
\pi_{i}^{\tau}\left(X_{i}^{0}+\tau, X_{A i}\left(X_{i}^{0}+\tau, X_{-i}^{0}-\tau\right)\right)+\pi_{-i}^{\tau}\left(X_{-i}^{0}-\tau, X_{A(-i)}\left(X_{i}^{0}+\tau, X_{-i}^{0}-\tau\right)\right) .
\end{gathered}
$$

\footnotetext{
${ }^{13}$ We abstract away from issues concerning the precise ex post division of the alliance's joint payoff. For cooperative game theoretic approaches to the theory of alliance costs and benefits see Sandler (1999) and Arce M. and Sandler (2001).
} 
In Case 2 this equals $\phi_{-i}-\frac{\phi_{-i}}{2\left(X_{-i}^{0}-\tau\right)}+\left(\frac{\phi_{i} \phi_{-i}\left(X_{i}^{0}+\tau\right)}{X_{-i}^{0}-\tau}\right)^{\frac{1}{2}}$. It follows directly that $\frac{\partial \pi_{12}^{\tau}}{\partial \tau}>0$ for all $\tau>0$ such that $\frac{X_{i}^{0}+\tau}{\phi_{i}}<\frac{X_{-i}^{0}-\tau}{\phi_{-i}}$. Thus, the alliance can strictly increase its payoff by diverting resources from Blotto game $-i$ to Blotto game $i$ up until the point at which $\frac{\phi_{i}}{\phi_{-i}}=\frac{X_{i}^{t}}{X_{-i}^{t}}$, as in Case 4.

In Case 4 , the payoff to the alliance is $\left(\phi_{1}+\phi_{2}\right)\left(1-\frac{1}{2 \hat{X}}\right)$. Given the arguments given above concerning Cases 1 and 2, it is clear that their are no profitable deviations for the alliance.

Lastly, in the case of an alliance with complete commitment and $\hat{X}<1$, the allocation of the alliance budget to the two Colonel Blotto games may satisfy the conditions for Case 2 or Case 3 (see Figure 4). Given the above arguments, any allocation by the alliance that satisfies the conditions for Case 2 is not an equilibrium strategy. In Case 3 the payoff of the alliance given any initial allocation, $\left(X_{i}^{0}, X_{-i}^{0}\right)$ in Case 3 and any transfer $\tau>0$ such that $\frac{X_{i}^{0}+\tau}{\phi_{i}} \leq \frac{X_{-i}^{0}-\tau}{\phi_{-i}}$, is given by

$$
\begin{gathered}
\pi_{12}^{\tau}\left(X_{i}^{0}+\tau, X_{-i}^{0}-\tau\right)= \\
\frac{\phi_{i}\left(X_{i}^{0}+\tau\right)}{2}+\frac{\phi_{-i}\left(X_{-i}^{0}-\tau\right)}{2}+\left(\phi_{i} \phi_{-i}\left(X_{-i}^{0}-\tau\right)\left(X_{i}^{0}+\tau\right)\right)^{\frac{1}{2}} .
\end{gathered}
$$

It follows directly that

$$
\frac{\partial \pi_{12}^{\tau}}{\partial \tau}=\frac{\phi_{i}}{2}-\frac{\phi_{-i}}{2}+\frac{1}{2}\left(\frac{\phi_{i} \phi_{-i}\left(X_{-i}^{0}-\tau\right)}{X_{i}^{0}+\tau}\right)^{\frac{1}{2}}-\frac{1}{2}\left(\frac{\phi_{i} \phi_{-i}\left(X_{i}^{0}+\tau\right)}{X_{-i}^{0}-\tau}\right)^{\frac{1}{2}}
$$

Solving for $\tau$ yields $\frac{\phi_{i}}{\phi_{-i}}=\frac{X_{i}^{0}+\tau}{X_{-i}^{0}-\tau}$, and thus $X_{i}^{t}=\frac{\hat{X} \phi_{i}}{\phi_{i}+\phi_{-i}}$ and $X_{-i}^{t}=\frac{\hat{X} \phi_{-i}}{\phi_{i}+\phi_{-i}}$. Q.E.D.

In contrast to the restricted range of initial endowments for which transfers take place in self-enforcing alliances without commitment, such transfers take place almost everywhere under alliances with complete commitment. Only when $X_{-i}^{0}=\frac{\phi_{-i}}{\phi_{i}} X_{i}^{0}$ does no transfer take place. However, as shown in panel (b) of Figure 5, there exist initial endowments for which 
a self-enforcing alliance without commitment yields the same outcome as under complete commitment, $X_{-i}^{t}=\frac{\phi_{-i}}{\phi_{i}} X_{i}^{t}$. This arises for a subset of the range of endowments in which the alliance member $\left(-i\right.$ in the figure), with the higher Colonel Blotto game value $\left(\phi_{-i}\right)$ has an endowment, $X_{-i}^{0}$, both larger than that of player $A$ and larger than the product of the ratio of game values and the alliance partner's endowment $\left(\frac{\phi_{-i}}{\phi_{i}} X_{i}^{0}\right)$.

Of course these two types of alliances form the two endpoints of the entire spectrum of possible levels of commitment. However, one might conjecture that intermediate levels of commitment generate regions of initial endowments where nonzero transfers take place that are nested between the regions corresponding to these two extremes.

\section{Conclusion}

The literature on the economics of alliances, originating with Olson (1965) and Olson and Zeckhauser (1966) focuses on the case where defense expenditures are a (possibly impure) public good and the threat of attack is exogenous. This paper extends this literature by examining the formation of self-enforcing alliances without commitment in a multi-player, multi-front Colonel Blotto game. In this case, the payoff to each alliance member is completely excludable and rival. Moreover, the common opponent is able to observe and react to the formation of the alliance and the resulting transfer of resources. Remarkably, we find that self-enforcing alliances without commitment form for a wide range of parameters. With ex ante asymmetry of resources - both in absolute terms and relative to the respective values at stake in the allies' Colonel Blotto games - a unilateral transfer from the relatively resource-rich ally to the relatively resource-poor ally causes a reallocation of the common opponent's resources that creates a strategic externality. The combination of the direct and strategic effects of such a transfer benefits both allies. For the ally making the transfer, the

positive strategic effect of the opponent's reallocation of resources away from their Blotto 
game makes up for the negative direct effect of the reduction in own resources. For the ally receiving the transfer, the positive direct effect of greater resource availability dominates the negative strategic effect of a higher opponent resource level.

Potential extensions of the model include the analysis of a more general network structure of battlefield alignment in which players may be engaged in several conflicts with different sets of adversaries, who may themselves be engaged in other conflicts. In this context, it is possible to carry out a nontrivial examination of the nature of the alliances that form and the composition of their membership. Our model also provides a useful tool for examining the strategic effects of precommitment to budgetary transparency. Since the payoffs and strategies in any Blotto game are parameterized by the players' budgets, our model is a natural framework for examining the costs and benefits of finer or coarser budgetary information and the effects of budgetary aggregation and disaggregation in entities engaged in conflict. It may also serve as a useful framework for the study of espionage.

Finally, although the analysis in this paper is framed in the context of defense or military alliances between nations, it is readily adapted to other contexts. For instance, in the context of multiple-product $\mathrm{R} \& \mathrm{D}$ races or patent races, it can be applied to explain research joint ventures and silent cross-industry partnerships ("cash infusions") between firms that do not compete in the same market, but face a common conglomerate competitor.

\section{References}

Arce M., D.G., and T. Sandler (2001) "A cooperative game theory of noncontiguous allies" Journal of Public Economic Theory 3, 391-411.

Bellman, R. (1969) "On Colonel Blotto and analogous games" Siam Review 11, 66-68.

Blackett, D.W. (1954) "Some Blotto games" Naval Research Logistics Quarterly 1, 55-60. 
Blackett, D.W. (1958) "Pure strategy solutions to Blotto games" Naval Research Logistics Quarterly 5, 107-109.

Bloch, F. (2010) "Endogenous formation of alliances in conflicts" in Handbook of the Economics of Peace and Conflict by Garfinkel, M., and S. Skaperdas, Eds., Oxford University Press: Oxford.

Borel, E. (1921) "La théorie du jeu les équations intégrales à noyau symétrique" Comptes Rendus de l'Académie 173, 1304-1308; English translation by Savage, L. (1953) "The theory of play and integral equations with skew symmetric kernels" Econometrica 21, $97-100$.

Borel, E., and J. Ville (1938) Application de la théorie des probabilitiés aux jeux de hasard, Gauthier-Villars: Paris; reprinted in Borel, E., and A. Chéron (1991) Théorie mathematique du bridge à la portée de tous, Editions Jacques Gabay: Paris.

Bruce, N. (1990) "Defence expenditures by countries in allied and adversarial relationships" Defence Economics 1, 179-195.

Cingranelli, D.L., and T.E. Pasquarello (1985) "Human rights practices and the distribution of U.S. foreign aid to Latin American countries" American Journal of Political Science 29, 539-563.

Golman, R., and S.E. Page (2009) "General Blotto: games of strategic allocative mismatch" Public Choice 138, 279-299.

Gross, O. (1951) "The symmetric Blotto game" RM-718, RAND Corporation, Santa Monica.

Gross, O., and R. Wagner (1950) "A continuous Colonel Blotto game" RM-408, RAND Corporation, Santa Monica. 
Hart, S. (2008) "Discrete Colonel Blotto and general lotto games" International Journal of Game Theory 36, 441-460.

Herring, G.C. Jr. (1973) Aid to Russia 1941-1946: Strategy, Diplomacy, the Origins of the Cold War, Columbia University Press: New York.

Hoey, M. (2009) "India's quest for dual-use technology" Bulletin of the Atomic Scientists 65, 43-59.

Hortala-Vallve, R., and A. Llorente-Saguer (2010a) "A simple mechanism for resolving conflict" Games and Economic Behavior in press, doi:10.1016/j.geb.2010.02.005.

Hortala-Vallve, R., and A. Llorente-Saguer (2010b) "Pure-strategy Nash equilibria in nonzero sum Colonel Blotto games" London School of Economics, mimeo.

Ihori, T., and M.C. McGuire (2007) "Collective risk control and group security: the unexpected consequences of differential risk aversion" Journal of Public Economic Theory 9, 231-263.

Konrad, K.A. (2009) Strategy and Dynamics in Contests, Oxford University Press: Oxford.

Kovenock, D., and B. Roberson (2010) "The optimal defense of networks of targets" Purdue University Economics Working Paper no. 1251.

Kvasov, D. (2007) "Contests with limited resources" Journal of Economic Theory 127, 738-748.

Laslier, J.F. (2002) "How two-party competition treats minorities" Review of Economic Design 7, 297-307.

Laslier, J.F., and N. Picard (2002) "Distributive politics and electoral competition" Journal of Economic Theory 103, 106-130. 
Linster, B.G. (1993) "A rent-seeking model of international competition and alliances" Defence Economics 4, 213-226.

Macdonell, S., and N. Mastronardi (2010) "Colonel Blotto equilibria: a complete characterization in the two battlefield case" University of Texas, mimeo.

Meernik, J., Krueger, E.L., and S.C. Poe (1998) "Testing models of U.S. foreign policy: foreign aid during and after the Cold War" Journal of Politics 60, 63-85.

Murdoch, J.C., and T. Sandler (1982) "A theoretical and empirical analysis of NATO" Journal of Conflict Resolution 26, 237-263.

Murdoch, J.C., and T. Sandler (1984) "Complementarity, free riding, and the military expenditures of NATO allies" Journal of Public Economics 25, 83-101.

Olson, M. (1965) The Logic of Collective Action, Harvard University Press: Cambridge, MA.

Olson, M., and R. Zeckhauser (1966) "An economic theory of alliances" Review of Economic Statistics 48, 266-279.

Roberson, B. (2006) "The Colonel Blotto game" Economic Theory 29, 1-24.

Roberson, B. (2008) "Pork-barrel politics, targetable policies, and fiscal federalism" Journal of the European Economic Association 6, 819-844.

Roberson, B., and D. Kvasov (2008) "The non-constant-sum Colonel Blotto game" CESifo Working Paper no. 2378.

Sandler, T. (1977) "Impurity of defense: an application to the economics of alliances" Kyklos 30, 443-460. 
Sandler, T. (1999) "Alliance formation, alliance expansion, and the core" Journal of Conflict Resolution 43, 727-747.

Sandler, T., and J. Cauley (1975) "On the economic theory of alliances" Journal of Conflict Resolution 19, 330-348.

Sandler, T., and K. Hartley (2001) "Economics of alliances: the lessons for collective action" Journal of Economic Literature 39, 869-896.

Skaperdas, S. (1998) "On the formation of alliances in conflict and contests" Public Choice 96, $25-42$.

Szentes, B., and R.W. Rosenthal (2003a) "Three-object two-bidder simultaneous auctions: chopsticks and tetrahedra" Games and Economic Behavior 44, 114-133.

Szentes, B., and R.W. Rosenthal (2003b) "Beyond chopsticks: symmetric equilibria in majority auction games" Games and Economic Behavior 45, 278-295.

Tang, P., Shoham, Y., and F. Lin (2010) "Designing competitions between teams of individuals" Artificial Intelligence 174, 749-766.

Tukey, J.W. (1949) "A problem of strategy" Econometrica 17, 73.

Van Ypersele De Strihou, J. (1967) "Sharing the defense burden among Western allies" Review of Economics and Statistics 49, 527-536.

Weinstein, J. (2005) "Two notes on the Blotto game" Northwestern University, mimeo. 


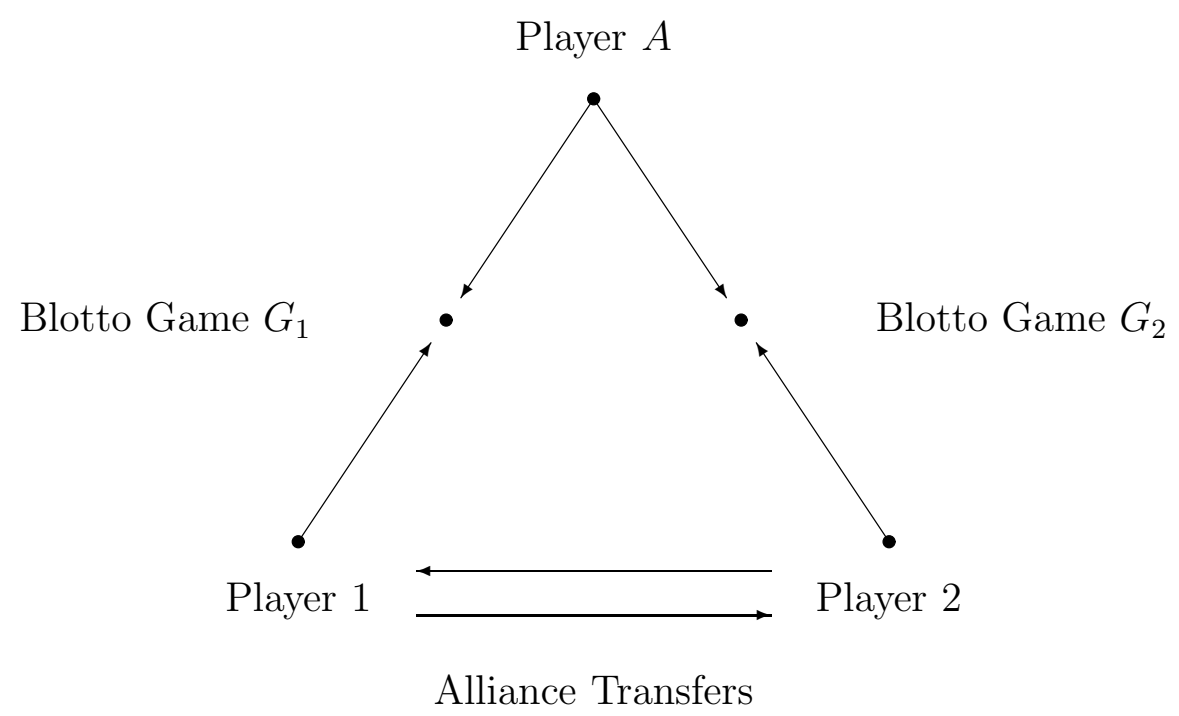

Figure 1: Coalitional Colonel Blotto Game Schematic 


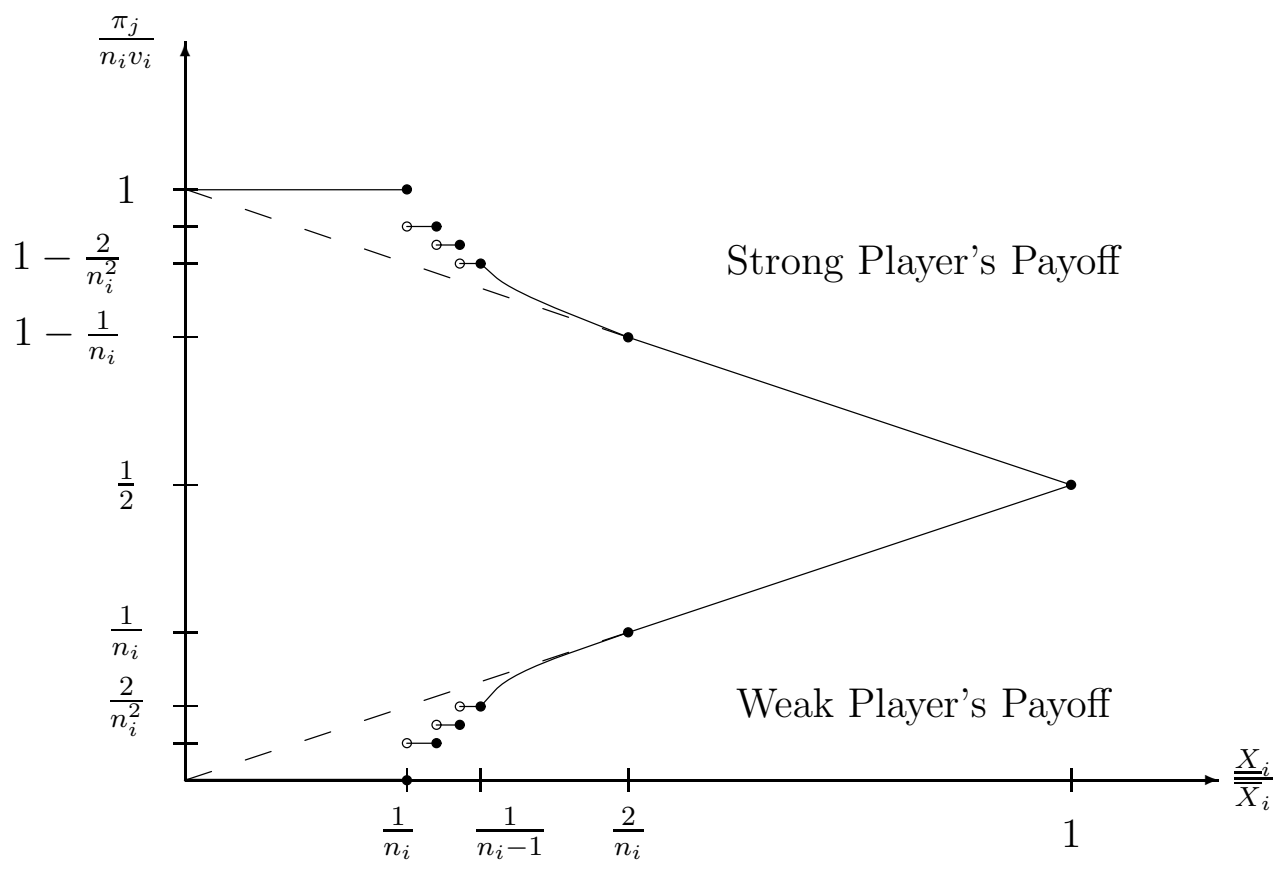

Figure 2: Payoffs in Colonel Blotto Game $i$ 

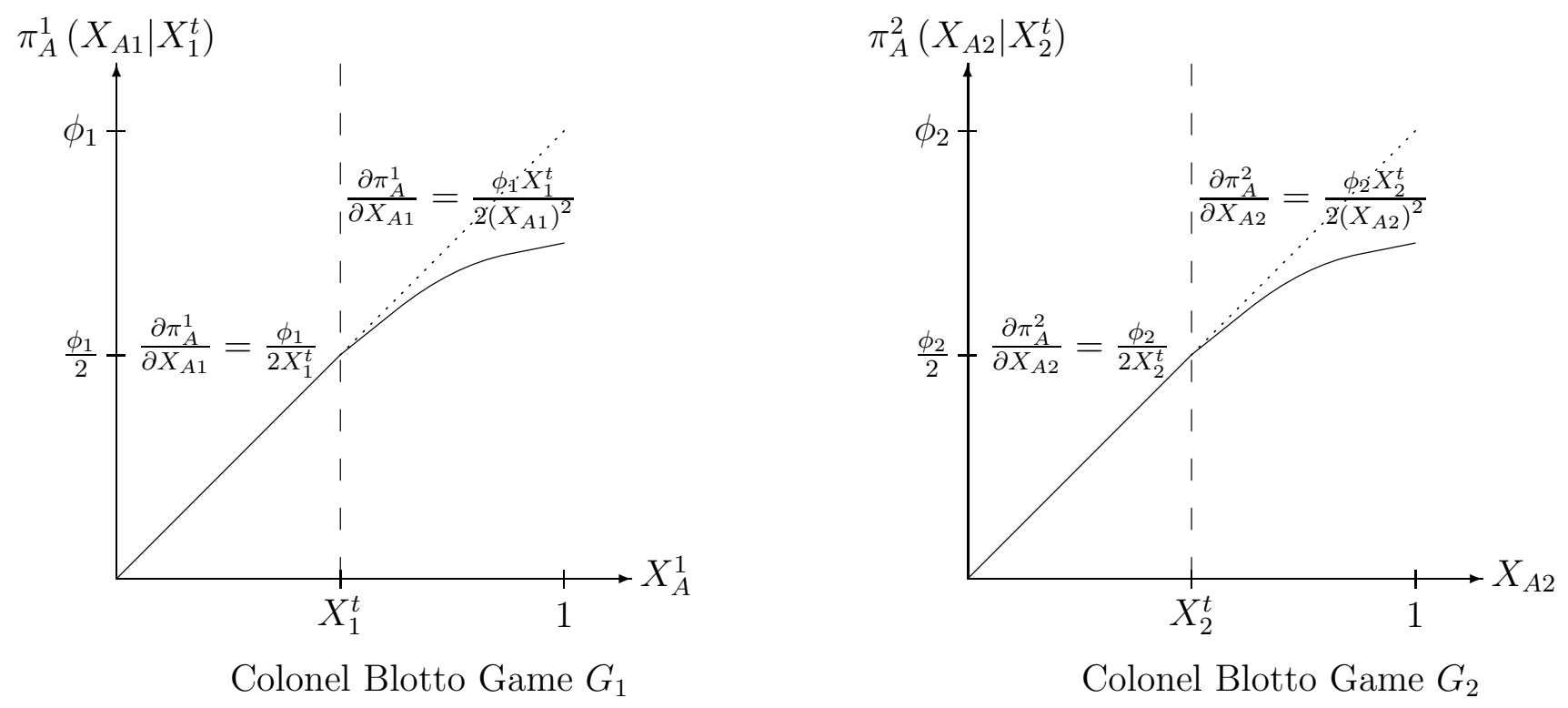

Figure 3: Player A's Payoffs in Each Colonel Blotto Game Given Players 1 and 2's Resource Levels 


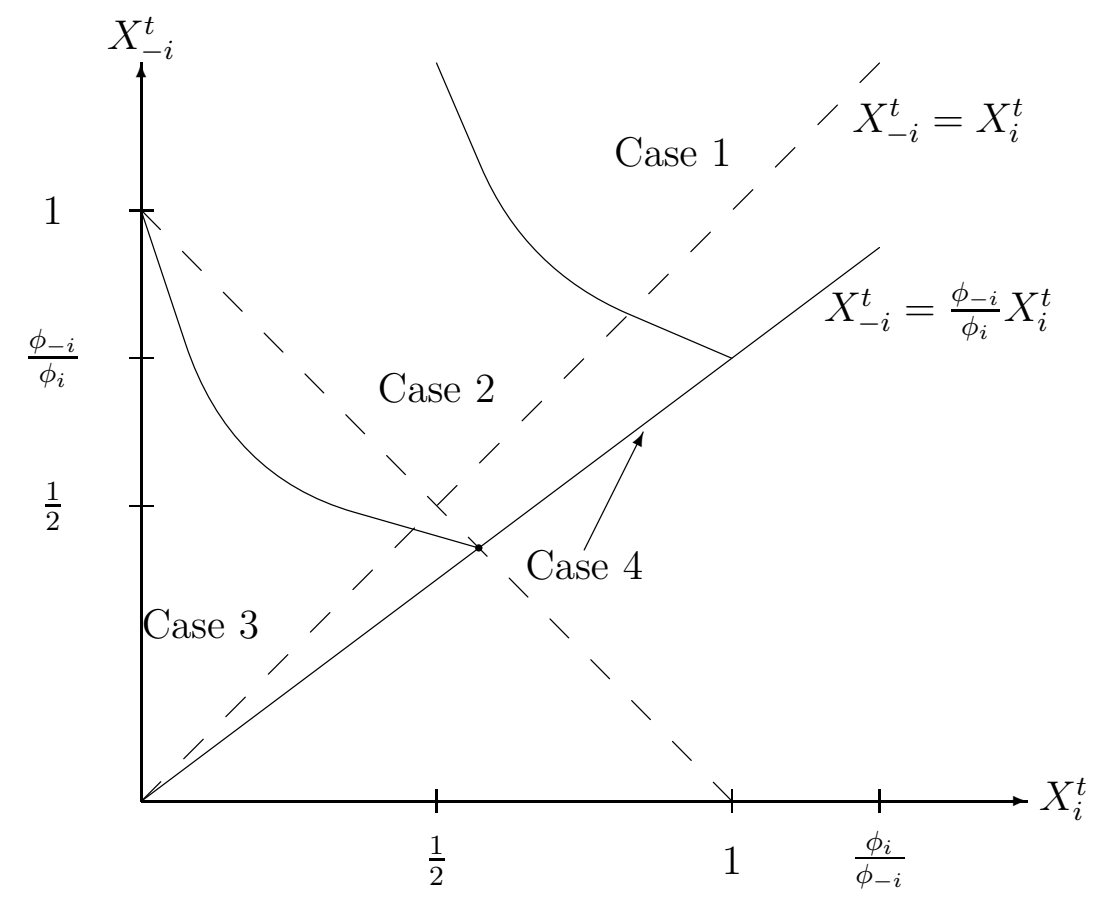

Figure 4: Cases of $A$ 's Optimal Resource Allocation $\left(\frac{\phi_{i}}{\phi_{-i}}>1\right)$ 


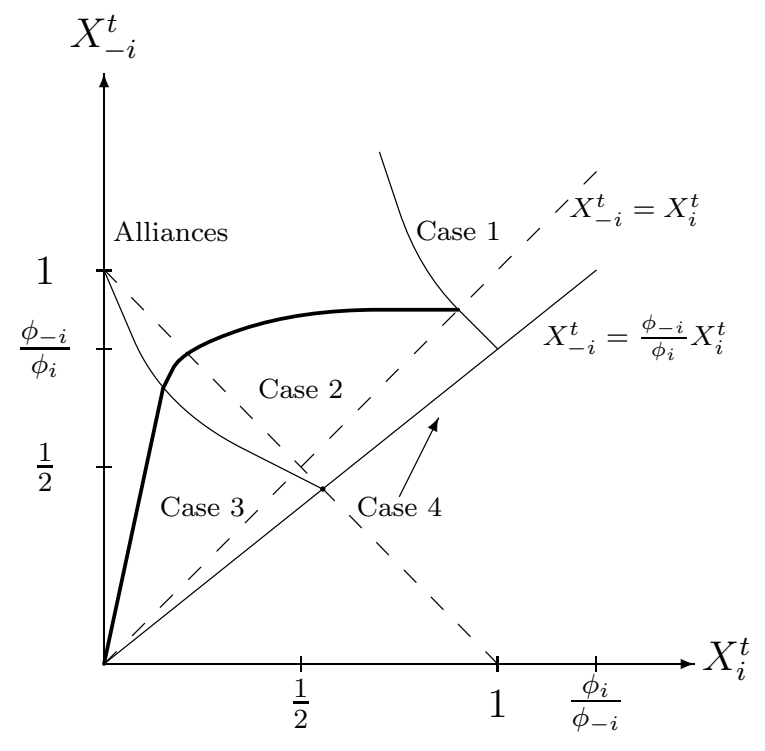

(a) $\frac{\phi_{-i}}{\phi_{i}}<1$

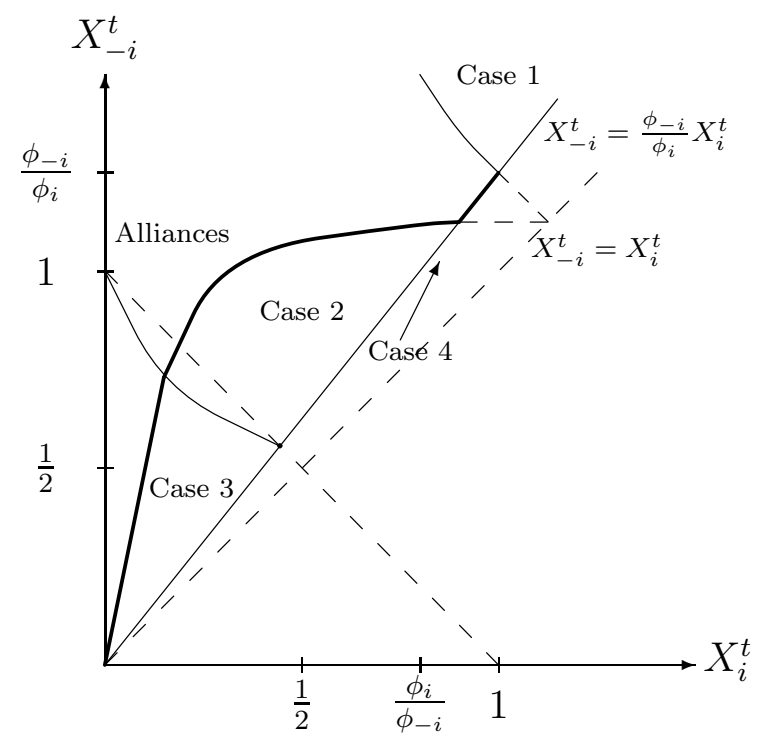

(b) $\frac{\phi_{-i}}{\phi_{i}}>1$

Figure 5: Ranges of Alliance Formation 